\title{
Three-Dimensional Metrics as Deformations of a Constant Curvature Metric
}

\author{
B. Coll $\stackrel{*}{,}$ J. Llosa ${ }^{\dagger}$ and D. Soler
}

July 23, 2021

\begin{abstract}
Any three-dimensional metric $g$ may be locally obtained from a constant curvature metric, $h$, by a deformation like

$$
g=\sigma h+\epsilon s \otimes s,
$$

where $\sigma$ and $s$ are respectively a scalar and a one-form, the sign $\epsilon= \pm 1$ and a functional relation between $\sigma$ and the Riemannian norm of $s$ can be arbitrarily prescribed. The general interest of this result in geometry and physics, and the related open problems, are stressed.
\end{abstract}

PACS number: 0420C

\section{Introduction}

It is known, since an old result by Riemann [1], that a $n$-dimensional metric has $f=n(n-1) / 2$ degrees of freedom, that is, it is locally equivalent to the giving of $f$ functions. As this feature is related to some particular choices of local charts, which are obviously non-geometric objects [2], it seems to be generically a not covariant property.

According to it, a two-dimensional metric has $f=1$ degrees of freedom. In this case, however, a stronger result holds, as it is well known [3], namely: any two-dimensional metric $g$ is locally conformally flat, $g=\phi \eta$, $\phi$ being the conformal deformation factor and $\eta$ the flat metric.

Contrarily to what the above Riemann's general result suggests, the twodimensional case is intrinsic and covariant, i.e. it only needs the knowledge of the metric $g$ and only involves tensor quantities, specifically, the sole degree of freedom is represented by a scalar, the conformal deformation factor $\phi$.

\footnotetext{
*Département d'Astronomie Fondamentale UMR-8630 CNRS, Observatoire de Paris, 61 Av. Observatoire; F-75014 Paris, France

${ }^{\dagger}$ Departament de Física Fonamental, Universitat de Barcelona, Diagonal, 647, E-08028 Barcelona, Spain; e-mail address: pitu@ffn.ub.es
} 
The question thus arises of, whether or not, for $n>2$ there exist similar intrinsic and covariant local relations between an arbitrary metric $g$, on the one hand, and the corresponding flat one $\eta$ together with a set of $f$ covariant quantities on the other.

To our knowledge, no result of this type has been published. Indeed, the known results concerning the diagonalization of any three-dimensional metric [4, 河] do not belong to this type. As a matter of fact, besides the $f=3$ scalars and the (more o less implicit) flat metric, these results also involve a particular ortogonal triad of vector fields. Also, in the context of the General Theory of Relativity, such a $n$-dimensional relation has been proposed by one of us, but unfortunately it remains for the moment only a mere conjecture.

In this paper we shall answer affirmatively the three-dimensional case. This dimension is the solution to the equation $f=n$, so that one is tempted to take (the components of) a vector field as the covariant set (of $f=3$ quantities). On the other hand, the result being deliberately local, it would seem that the essentials of the flat metric in this matter is its minimal freedom, i.e. the maximal dimension of its isometry group, so that it should be possible to substitute it by a prescribed constant curvature metric. We shall see that both assumptions work.

In fact, the paper is devoted to prove the following main result:

Theorem 1 Any three-dimensional Riemannian metric $g$ may be locally obtained from a constant curvature metric $h$ by a deformation of the form

$$
g=\sigma h+\epsilon \boldsymbol{s} \otimes \boldsymbol{s},
$$

where $\sigma$ and $s$ are respectively a scalar function and a differential 1-form; the sign $\epsilon= \pm 1$ and a relationship $\Psi(\sigma,|s|)$ between the scalar $\sigma$ and the Riemannian norm $|s|$ may be arbitrarily prescribed.

This result should be interesting in geometrical as well as in physical situations.

In geometry, perhaps one of the first questions to be answered is the following: In two dimensions it is known that the gauge of the conformal factor $\sigma$ or, equivalently, the set of flat metric tensors conformal to a given metric is given by the solutions of the Laplacian, $\Delta \sigma=0$ [6]. In the three-dimensional case here considered, what is the gauge of the vector fields $s$ associated to a given metric $g$ ? or, equivalenty, how many constant curvature metric tensors $h$ correspond to $g$ through the relation (11)?

But many other interesting questions arise. For instance, since the theorem states a correspondence between a metric $g$ and a couple $(\sigma, s$, what conditions must $\sigma$ and $s$ fulfill in order that $g$ admits a continuous group of symmetries?

In classical physics, the above theorem should be useful in (finite) deformation theory of materials; equation (11) may be considered as an ideal universal deformation law, allowing, from an unconstrained or not initial state (described in material coordinates by the tensor $h$ ), to reach any other deformation state (described in the same coordinates by the tensor $g$ ) [7]. This ideal universal 
law allows to associate, to every deformation state of a material, a vector field $s$ among those of the gauge class of the flat metric.

In general relativity, any vacuum space-time is locally equivalent to its Cauchy data, $\{g, K\}, g$ being the spatial metric and $K$ the extrinsic curvature of the initial instant. These data have to verify the constraint equations, a set of four equations for which many years ago Lichnerowicz showed [8] that to every arbitrarily given metric $\tilde{g}$ it corresponds a unique solution $\{g, K\}$ such that $g=\sigma \tilde{g}$. This beautiful result is however useless for precise physical situations because, $g$ being initialy unknown, one does not see how to choose the good starting metric $\tilde{g}$, which has to give $g$ by conformity. Such an objection may be eliminated using (1i) in the constraint equations. Our theorem also allows to translate notions such as asymptotic flatness or spatial singularity in terms of the differential 1-form $s$ over a flat metric $h$.

The paper is organized as follows. Sections 2 and 3 are devoted to proof the above theorem and some examples of this result are presented in section 4 .

\section{Flat deformation of a given metric}

Instead of proving theorem 1 as stated in the introduction, we shall prove the following equivalent result:

Theorem 2 Let $(\mathcal{V}, g)$ be a Riemannian 3-manifold. There locally exist a function $\phi$ and a differential 1-form $\mu$ such that the tensor

$$
\tilde{g}:=\phi g-\epsilon \boldsymbol{\mu} \otimes \boldsymbol{\mu}
$$

(with $\epsilon= \pm 1$ ) is also a Riemannian metric with constant curvature. Besides, an arbitrary relation between $\phi$ and $|\mu|^{2}:=g^{i j} \mu_{i} \mu_{j}$ can be imposed in advance.

The equivalence between both theorems follows immediately on substituting

$$
h=\tilde{g}, \quad \sigma=\phi^{-1}, \quad s=\phi^{-1 / 2} \boldsymbol{\mu}
$$

into equation (11). The present formulation (2) stresses that we seek to derive $\tilde{g}$ from a given $g$.

The proof spreads over sections 2 and 3 and is based on the comparison of the Riemannian geometries respectively defined by $g$ and $\tilde{g}$.

We start by considering the Riemannian connexions $\nabla$ and $\tilde{\nabla}$. In an arbitrary frame $\left\{e_{i}\right\}_{i=1,2,3}$ the expression (2) reads:

$$
\tilde{g}_{i j}:=\phi g_{i j}-M_{i j}, \quad \text { with } \quad M_{i j}:=\epsilon \mu_{i} \mu_{j}
$$

We shall consider the difference tensor:

$$
B_{k i}^{j}:=\tilde{\gamma}_{k i}^{j}-\gamma_{k i}^{j}
$$

which is symmetric:

$$
B_{k i}^{j}=B_{i k}^{j}
$$


because both connexions are torsion free.

Now, since $\nabla_{k} g_{i j}=\tilde{\nabla}_{k} \tilde{g}_{i j}=0$ and taking (5) into account, we easily obtain that:

$$
B_{i k}^{j}=\frac{1}{2}\left[\phi_{k} g_{i r}+\phi_{i} g_{k r}-\phi_{r} g_{i k}-\nabla_{k} M_{i r}-\nabla_{i} M_{k r}+\nabla_{r} M_{i k}\right] \tilde{h}^{r j}
$$

where

$$
\tilde{h}^{r j}:=\phi^{-1}\left(g^{r j}+\frac{1}{\phi-m_{0}} M^{r j}\right), \quad \text { with } \quad m_{0}:=g^{i j} M_{i j}=\epsilon|\mu|^{2},
$$

is the inverse metric for $\tilde{g}_{i j}$. (Indices are always raised, resp., lowered, with the metric $g^{i j}$, resp., $g_{i j}$, and the notation $\tilde{g}^{r j}$ is reserved to $\tilde{g}_{i l} g^{i r} g^{l j}$.)

\subsection{The curvature tensors}

The curvature tensor for $\tilde{g}$ is 9

$$
\tilde{R}_{i k l}^{j}=e_{k} \tilde{\gamma}_{l i}^{j}-e_{l} \tilde{\gamma}_{k i}^{j}+\tilde{\gamma}_{k m}^{j} \tilde{\gamma}_{l i}^{m}-\tilde{\gamma}_{l m}^{j} \tilde{\gamma}_{k i}^{m}-c_{k l}^{m} \tilde{\gamma}_{m i}^{j}
$$

For a 3-manifold this tensor is equivalent to the tensor (the one can be obtained from the other)

$$
\begin{aligned}
\tilde{G}^{i j} & =\frac{1}{4} \tilde{\eta}^{i k l} \tilde{\eta}^{j s r} \tilde{R}_{s r k l} \\
& =\frac{1}{2} \tilde{\eta}^{i k l} \tilde{\eta}_{s}^{j}{ }^{r}\left(e_{k} \tilde{\gamma}_{l r}^{s}+\tilde{\gamma}_{k m}^{s} \tilde{\gamma}_{l r}^{m}-\tilde{\gamma}_{k l}^{m} \tilde{\gamma}_{m r}^{s}\right)
\end{aligned}
$$

where $\tilde{\eta}^{i k l}$ is the contravariant volume tensor associated to $\tilde{g}$. This $\tilde{G}^{i j}$ is related to the Einstein tensor. Indeed, in three dimensions [10]:

$$
\tilde{R}_{s r k l}=\tilde{g}_{r k} \tilde{R}_{s l}+\tilde{g}_{s l} \tilde{R}_{r k}-\tilde{g}_{r l} \tilde{R}_{i j}-\tilde{g}_{s k} \tilde{R}_{r l}+\frac{\tilde{R}}{2}\left(\tilde{g}_{s k} \tilde{g}_{r l}-\tilde{g}_{s l} \tilde{g}_{r k}\right)
$$

whence it follows immediately that: $\tilde{G}^{i j}=\tilde{R}^{i j}-\frac{1}{2} \tilde{h}^{i j} \tilde{R}$.

Similar relations hold for the curvature tensor $R^{j}{ }_{i k l}$, the metric $g_{i j}$ and the volume tensor $\eta^{i k l}$.

Using equations (9) and (14), and the fact that the volume tensors $\tilde{\eta}^{i k l}$ and $\eta^{i k l}$ are proportional - see appendix $\mathrm{A}$ - we obtain:

$$
\tilde{G}^{i j}=D^{2} G^{i j}+\frac{D^{2}}{2} \eta^{i k l} \eta_{s}^{j}{ }^{r}\left(\nabla_{k} B_{l r}^{s}+B_{k m}^{s} B_{l r}^{m}\right)
$$

where the relationships (36) and (37) in Appendix A:

$$
\tilde{\eta}^{i k l}=D \eta^{i k l}, \quad D^{2}:=\frac{\operatorname{det} g}{\operatorname{det} \tilde{g}}=\frac{\phi^{-2}}{\phi-m_{0}}
$$

have been taken into account. 
The condition that $\tilde{g}$ has constant curvature [1] is:

$$
\tilde{R}_{j i k l}=K\left(\tilde{g}_{j k} \tilde{g}_{i l}-\tilde{g}_{j l} \tilde{g}_{i k}\right), \quad K=\text { constant },
$$

which in terms of $\tilde{G}^{i j}$ reads:

$$
\tilde{H}^{i j}:=\tilde{G}^{i j}-K \tilde{h}^{i j}=0
$$

\subsection{The second Bianchi identity}

In terms of the tensor $\tilde{G}^{i j}$, the second Bianchi identity [12] reads

$$
\tilde{\nabla}_{i} \tilde{G}^{i j} \equiv 0
$$

which, using the tensor $\tilde{H}^{i j}$ introduced above and taking into account that $K$ is a constant, leads to:

$$
\tilde{\nabla}_{i} \tilde{H}^{i j} \equiv 0 ;
$$

In terms of the connexion $\nabla$ and the difference tensor $B_{i k}^{j}$, this identity can be also written as:

$$
\nabla_{i} \tilde{H}^{i j}+B_{i l}^{i} \tilde{H}^{l j}+B_{i l}^{j} \tilde{H}^{l i} \equiv 0
$$

and must be understood as follows:

Lemma 1 Let $g, \phi$ and $M_{i j}$ be, respectively, a metric, a scalar function and a symmetric tensor such that $\tilde{g}$ defined by (3) is regular. Then the tensor field $\tilde{G}^{i j}(g, \gamma, \phi, M)$ defined by (10) satisfies identically (13).

In the next section we shall consider the condition (11) as a partial differential system on the unknowns $\boldsymbol{\mu}_{i}$ and $\phi$. The problem of solving this system is pretty similar to solve Einstein equations in 3 dimensions.

\section{The Cauchy problem}

Let $\mathcal{S}_{0}$ be a surface in a 1-parameter family of surfaces $\mathcal{S}_{\lambda} \subset \mathcal{V}_{3}$, let $n$ be the unit $g$-normal vector and let $\left\{e_{i}\right\}_{i=1,2,3}$ be a $g$-orthonormal tetrad adapted to $\mathcal{S}_{\lambda}$, i. e., $e_{3}=n$.

We have to solve the second order partial differential system (11) with $\tilde{G}^{i j}$ given by (10). We first notice that the set of three equations:

$$
\tilde{H}^{3 j}:=\tilde{G}^{3 j}-K \tilde{h}^{3 j}=0, \quad j=1,2,3
$$

do not contain second order normal derivatives of the unknowns: neither $\nabla_{3} \nabla_{3} M_{k l}$ nor $\nabla_{3} \nabla_{3} \phi$. Indeed,

$$
\eta^{3 k l} \nabla_{k}=\sum_{b=1}^{2} \eta^{3 b l} \nabla_{b}
$$

only involves tangential derivatives, i. e., along $e_{1}$ and $e_{2}$. (Hereon the indices $a, b, c, \ldots$ run from 1 to 2 , whereas the indices $i, j, k, \ldots$ run from 1 to 3 .) 
On the other hand, the remaining three equations:

$$
\tilde{H}^{a b}:=\tilde{G}^{a b}-K \tilde{h}^{a b}=0, \quad a, b=1,2
$$

do contain second order normal derivatives. After a short calculation, taking (3), (6), (7) and (10) into account, we readily obtain that eq. (15) can be written as:

$$
\tilde{H}^{a b}:=\frac{D^{2}}{4} \epsilon^{a 3 l} \epsilon_{b s r} \tilde{h}_{s}^{j}\left(\delta_{r}^{3} \delta_{j}^{i}-\delta_{j}^{3} \delta_{r}^{i}\right)\left(\ddot{\phi} \delta_{i l}-\epsilon \boldsymbol{\mu}_{i} \ddot{\boldsymbol{\mu}}_{i}-\epsilon \boldsymbol{\mu}_{l} \ddot{\boldsymbol{\mu}}_{i}\right)+P^{a b}=0
$$

where a dot means the covariant normal derivative $\nabla_{3}$, and $P^{a b}$ does not depend on second order normal derivatives.

We have however three equations and four unknowns, hence the problem is, at this stage, underdetermined. We can thus introduce an arbitrary additional relation:

$$
\Psi\left(\phi, m_{0}\right)=0
$$

which will be hereafter referred to as gauge.

By successive differentiation along $\nabla_{3}$, this constraint induces other differential constraints, namely,

$$
\begin{aligned}
\nabla_{3} \Psi & :=\Psi_{1} \dot{\phi}+\Psi_{2} 2 \epsilon \boldsymbol{\mu}^{i} \dot{\boldsymbol{\mu}}_{i}=0 \\
\nabla_{3} \nabla_{3} \Psi & :=\Psi_{1} \ddot{\phi}+\Psi_{2} 2 \epsilon \boldsymbol{\mu}^{i} \ddot{\boldsymbol{\mu}}_{i}+P_{0}=0
\end{aligned}
$$

where:

$$
\Psi_{1}:=\frac{\partial \Psi}{\partial \phi} \quad \text { and } \quad \Psi_{2}:=\frac{\partial \Psi}{\partial m_{0}} .
$$

and $P_{0}$ does not depend on second order normal derivatives.

Now, the second order partial differential system

$$
\left.\begin{array}{l}
\tilde{H}^{a b}=0, \quad a, b=1,2 \\
\nabla_{3} \nabla_{3} \Psi=0
\end{array}\right\}
$$

is quasilinear and has four equations for four unknowns.

Its characteristic determinant is:

$$
\Delta_{1}=\left|\begin{array}{cccc}
\frac{-D^{2}\left(2 \phi-m_{0}-\epsilon\left(\boldsymbol{\mu}_{1}\right)^{2}\right)}{4 \phi\left(-m_{0}\right)} & \frac{D^{2} \boldsymbol{\mu}_{1}\left(\boldsymbol{\mu}_{2}\right)^{2}}{4 \phi\left(\phi-m_{0}\right)} & \frac{D^{2} \epsilon \boldsymbol{\mu}_{2}\left(4 \phi-2 m_{0}-\epsilon\left(\boldsymbol{\mu}_{2}\right)^{2}\right)}{4 \phi\left(\phi-m_{0}\right)} & 0 \\
\frac{D^{2} \boldsymbol{\mu}_{1} \boldsymbol{\mu}_{2}}{4 \phi\left(\phi-m_{0}\right)} & -\frac{D^{2} \epsilon \boldsymbol{\mu}_{2}\left(2 \phi-m_{0}-\epsilon\left(\boldsymbol{\mu}_{2}\right)^{2}\right)}{4 \phi\left(\phi-m_{0}\right)} & -\frac{D^{2} \epsilon \boldsymbol{\mu}_{1}\left(2 \phi-m_{0}+\epsilon\left(\boldsymbol{\mu}_{2}\right)^{2}\right)}{4 \phi\left(\phi-m_{0}\right)} & 0 \\
-\frac{D^{2}\left(2 \phi-m_{0}-\epsilon\left(\boldsymbol{\mu}_{2}\right)^{2}\right)}{4 \phi\left(\phi-m_{0}\right)} & \frac{D^{2} \epsilon \boldsymbol{\mu}_{1}\left(4 \phi-2 m_{0}-\epsilon\left(\boldsymbol{\mu}_{2}\right)^{2}\right)}{4 \phi\left(\phi-m_{0}\right)} & \frac{D^{2} \boldsymbol{\mu}_{2}\left(\boldsymbol{\mu}_{1}\right)^{2}}{4 \phi\left(\phi-m_{0}\right)} & 0 \\
\Psi_{I} & 2 \epsilon \Psi_{2} \boldsymbol{\mu}_{1} & 2 \epsilon \Psi_{2} \boldsymbol{\mu}_{2} & 2 \epsilon \Psi_{2} \boldsymbol{\mu}_{3}
\end{array}\right|
$$

or

$$
\begin{aligned}
\Delta_{1}= & \frac{D^{6}}{2^{4}} \boldsymbol{\mu}_{3} \Psi_{2} \epsilon\left[\left(\boldsymbol{\mu}_{1}\right)^{2}+\left(\boldsymbol{\mu}_{2}\right)^{2}\right]\left[2 \phi-2 m_{0}+\epsilon\left(\boldsymbol{\mu}_{3}\right)^{2}\right] \\
& {\left[2 \phi-m_{0}-\epsilon\left(\boldsymbol{\mu}_{2}\right)^{2}\right]\left[2 \phi-m_{0}\right] }
\end{aligned}
$$


$\mathcal{S}_{0}$ is non-characteristic [13] if $\Delta_{1} \neq 0$ that, taking (38) into account (see the Appendix) — which ensures that both $g$ and $\tilde{g}$ are nondegenerate and positive-, reduces to:

$$
\Psi_{2}=\frac{\partial \Psi}{\partial m_{0}} \neq 0, \quad \boldsymbol{\mu}_{3} \neq 0 \quad \text { and } \quad \boldsymbol{\mu}_{1}^{2}+\boldsymbol{\mu}_{2}^{2} \neq 0
$$

As a consequence, we have shown the following result

Theorem 3 Let $\mathcal{S}_{0} \subset \mathcal{V}$ be a surface and $\left\{e_{i}\right\}_{i=1,2,3}$ a g-orthonormal frame adapted to $\mathcal{S}_{0}$, and let us be given:

(a) a gauge $\Psi\left(\phi, m_{0}\right)$ and

(b) a set of Cauchy data:

$$
\overline{\boldsymbol{\mu}_{i}}=\left.\boldsymbol{\mu}_{i}\right|_{\mathcal{S}_{0}}, \quad \bar{\phi}=\left.\phi\right|_{\mathcal{S}_{0}}, \quad \overline{\dot{\boldsymbol{\mu}}_{i}}=\left.\nabla_{3} \boldsymbol{\mu}_{i}\right|_{\mathcal{S}_{0}}, \quad \overline{\dot{\phi}}=\left.\nabla_{3} \phi\right|_{\mathcal{S}_{0}}
$$

(where a bar means "the value on $\mathcal{S}_{0}$ ) such that:

$$
\overline{\boldsymbol{\mu}_{3}} \neq 0, \quad \boldsymbol{\mu}_{1}^{2}+\boldsymbol{\mu}_{2}^{2} \neq 0, \quad \bar{\phi}>0, \quad \text { and } \bar{\phi}-\overline{m_{0}}>0,
$$

(S2) the gauge does depend on $m_{0}$, that is, $\left.\frac{\partial \Psi}{\partial m_{0}}\right|_{\mathcal{S}_{0}} \neq 0$

(S3) equations (18) and (19) hold on $\mathcal{S}_{0}$, and

(S4) the subsidiary conditions: $\left.\tilde{H}^{3 j}\right|_{\mathcal{S}_{0}}=0, j=1,2,3$

We can then find a solution $\boldsymbol{\mu}_{l}$, $\phi$ defined on a neighbourhood $\mathcal{U}$ of $\mathcal{S}_{0}$ such that fulfills (11) and (17), i. e.:

$$
\tilde{H}^{i j}\left(\phi, \boldsymbol{\mu}_{l}\right)=0, \quad i, j=1,2,3 \quad \text { and } \quad \Psi\left(\phi, m_{0}\right)=0 .
$$

Proof: Indeed, by conditions $\mathbf{S 1}$ and $\mathbf{S 2}$ above, $\mathcal{S}_{0}$ is non-characteristic, the Cauchy-Kovalevski theorem [13] can be applied and a solution $\phi, \boldsymbol{\mu}_{l}$ of the partial differential system (20) can be found in a neigbourhood $\mathcal{U}_{1}$ of $\mathcal{S}_{0}$ fulfilling conditions S1 through $\mathbf{S} 4$.

Now, let $\tilde{g}_{i j}$ be the metric constructed on $\mathcal{U}_{1}$ by substitution of the solution $\phi$ and $\boldsymbol{\mu}_{i}$ into (3). Let us see that $\tilde{g}_{i j}$ has constant curvature, i. e., $\tilde{H}^{i j}=0$, for $i, j=1,2,3$ on a neigbourhood of $\mathcal{S}_{0}$.

We have on the one hand that $\tilde{H}^{a b}=0$, for $a, b=1,2$, because $\phi$ and $\boldsymbol{\mu}_{i}$ is a solution of 20). And, on the other, the remaining three equations, namely, $\tilde{H}^{3 j}=0, j=1,2,3$, hold on $\mathcal{S}_{0}$ (condition S4).

To prove that the latter condition propagates well to a neigbourhood of $\mathcal{S}_{0}$, we separate the normal and tangential derivatives in the Bianchi identity (13) and, taking into account that (20) holds on $\mathcal{U}_{1}$, we arrive at:

$\nabla_{3} \tilde{H}^{3 j}+\sum_{b=1}^{2}\left[e_{b} \tilde{H}^{b 3} \delta_{3}^{j}+2 B_{3 b}^{j} \tilde{H}^{3 b}+\Gamma_{b 3}^{b} \tilde{H}^{3 j}+\Gamma_{b 3}^{j} \tilde{H}^{b 3}\right]+B_{i 3}^{i} \tilde{H}^{3 j}+B_{33}^{j} \tilde{H}^{33}=0$ 
The latter can be taken as a linear homogeneous partial differential system for the unknown $\tilde{H}^{3 j}$ which, for the Cauchy data expressed by condition $\mathbf{S} 4$ has the unique solution $\tilde{H}^{3 j}=0$ on a neigbourhood $\mathcal{U}_{2}$ of $\mathcal{S}_{0}$. Hence,

$$
\tilde{H}^{i j}=0, \quad \text { on } \quad \mathcal{U}=\mathcal{U}_{1} \cap \mathcal{U}_{2}
$$

and $\tilde{g}_{i j}$ has constant curvature in $\mathcal{U}$.

It is obvious that the gauge condition $\Psi\left(\phi, m_{0}\right)=0$ also propagates to the neigbourhood of $\mathcal{S}_{0}$, as a consequence of the last equation in (20) and the conditions S3.

\subsection{The subsidiary conditions}

We shall now see whether the subsidiary conditions (14) are not too restrictive. In the adapted $g$-orthonormal frame $\left\{e_{i}\right\}_{i=1,2,3}$ introduced at the begining of this section, the conditions (14) and the second of equations (18) read:

$$
\begin{gathered}
\eta^{3 a b} \nabla_{a}\left(B_{b r}^{s} \eta_{s}^{j}{ }^{r}\right)+\eta^{3 a b} \eta_{s}^{j}{ }^{r} B_{a m}^{s} B_{b r}^{m}+2 G^{3 j}-\frac{2 K}{D^{2}} \tilde{h}^{3 j} \approx 0 \\
\Psi_{I} \dot{\phi}+2 \Psi_{2} \boldsymbol{\mu}^{i} \dot{\boldsymbol{\mu}}_{i} \approx 0
\end{gathered}
$$

where " $\approx$ " means that the equality holds on $\mathcal{S}_{0}$.

The latter equations yield four relations to be fulfilled by the Cauchy data and can be used as a partial differential system on $\mathcal{S}_{0}$ to determine part of the Cauchy data, namely: $\bar{\phi}$ and $\overline{\dot{\boldsymbol{\mu}}_{i}}$, in terms of $\bar{\phi}$ and $\overline{\boldsymbol{\mu}_{i}}$.

Making explicit the terms containing $\nabla_{a} \overline{\dot{\phi}}$ and $\nabla_{a} \overline{\dot{\mu}_{i}}$, equations (24) and (25) respectively yield:

$$
A^{j a} \nabla_{a} \overline{\dot{\phi}}+A^{l j a} \overline{\dot{\boldsymbol{\mu}}_{l}}+Z^{j} \approx 0
$$

and

$$
2 \overline{\boldsymbol{\mu}^{i}} \Psi_{2} \nabla_{a} \overline{\dot{\boldsymbol{\mu}}_{i}}+\Psi_{I} \nabla_{a} \overline{\dot{\phi}}+Z \approx 0
$$

where $Z$ and $Z_{j}$ depend only on $\bar{\phi}$ and $\overline{\boldsymbol{\mu}_{i}}$, their derivatives tangential to $\mathcal{S}_{0}$ and on $\overline{\dot{\phi}}$ and $\overline{\dot{\boldsymbol{\mu}}_{i}}$, but not on tangential derivatives of the latter.

Furthermore, a short calculation yields:

$$
\begin{aligned}
A^{j a}= & \frac{1}{2 \bar{\phi}}\left[\left(2+\frac{\epsilon\left(\overline{\boldsymbol{\mu}_{3}}\right)^{2}}{\bar{\phi}-\bar{m}_{0}}\right) \delta^{j a}+\frac{\epsilon}{\bar{\phi}-\bar{m}_{0}} \bar{q}^{a} \bar{q}^{j}-\frac{\epsilon \overline{\boldsymbol{\mu}_{3}}}{\bar{\phi}-\bar{m}_{0}} \overline{\boldsymbol{\mu}^{a}} \delta_{3}^{j}\right] \\
A^{l j a}= & \frac{\epsilon}{2 \bar{\phi}}\left[2 \overline{\boldsymbol{\mu}^{a}} \eta^{l j 3}+\frac{\epsilon \overline{\boldsymbol{\mu}_{3}}}{\bar{\phi}-\bar{m}_{0}} \overline{\boldsymbol{\mu}^{a}} \eta^{l j s} \overline{\boldsymbol{\mu}_{s}}-\eta^{3 a l} \bar{q}^{j} \frac{2 \bar{\phi}-\bar{m}_{0}}{\bar{\phi}-\bar{m}_{0}}-\right. \\
& \left.\frac{\epsilon}{\bar{\phi}-\bar{m}_{0}} \bar{q}^{a} \bar{q}^{j} \overline{\boldsymbol{\mu}_{l}}\right]
\end{aligned}
$$

where $\bar{q}^{i}:=\eta^{3 i j} \overline{\boldsymbol{\mu}_{j}}$. 
To put the Cauchy problem for the partial differential system (26-27) on $\mathcal{S}_{0}$, let $\mathcal{C} \subset \mathcal{S}_{0}$ be a given curve and $\tau=\tau^{a} e_{a} \in T \mathcal{S}_{0}$, the unit vector orthogonal to $\mathcal{C}$. Assume that an adapted frame is chosen in $T \mathcal{S}_{0}$, such that $\tau^{1}=0$ and $\tau^{2}=1$, then the curve is non-characterisitc if, and only if, the characteristic determinant does not vanish, i. e.:

$$
\Delta_{2}:=-\frac{\epsilon{\overline{\mu_{2}}}^{2} \overline{\boldsymbol{\mu}}^{2} \overline{\boldsymbol{\mu}}^{2} \Psi_{2}\left[2 \bar{\phi}-2 \bar{m}_{0}+\epsilon \overline{\boldsymbol{\mu}}_{3}^{2}\right]\left(2 \bar{\phi}-\bar{m}_{0}\right)}{2 \bar{\phi}^{3}\left(\bar{\phi}-\bar{m}_{0}\right)^{3}} \neq 0
$$

That is, if, and only if, the data $\bar{\phi}, \overline{\boldsymbol{\mu}_{i}}$ on $\mathcal{S}_{0}$ are given such that:

$$
\begin{aligned}
& \Psi\left(\bar{\phi}, \bar{m}_{0}\right)=0 \quad \text { and } \quad \overline{\boldsymbol{\mu}_{i}} \neq 0, \quad i=1,2,3 \\
& \text { and } \frac{\partial \Psi}{\partial \bar{m}_{0}} \neq 0 \quad \text { on } \mathcal{S}_{0} .
\end{aligned}
$$

\subsection{Summary}

What we have proved so far is that for any given:

(a) surface $\mathcal{S}_{0}$ and curve $\mathcal{C} \in \mathcal{S}_{0}$,

(b) gauge function $\Psi\left(\phi, m_{0}\right)$ and

(c) data: $\bar{\phi}, \overline{\boldsymbol{\mu}_{i}}, i=1,2,3$ on $\mathcal{S}_{0}$ and $\overline{\dot{\phi}}, \overline{\dot{\boldsymbol{\mu}}_{i}}, i=1,2,3$ on $\mathcal{C}$, such that

$$
\Psi_{2}\left(\bar{\phi}, \bar{m}_{0}\right) \neq 0, \quad \bar{\phi}>0, \quad \bar{\phi}-\bar{m}_{0}>0 \text { and } \overline{\boldsymbol{\mu}_{j}} \neq 0, j=1,2,3
$$

the quasilinear partial differential system (26) and (27) can be integrated to determine $\overline{\dot{\phi}}$ and $\overline{\dot{\boldsymbol{\mu}}_{j}}, j=1,2,3$ on a surface $\mathcal{S}_{1}$ (a neighbourhood of $\mathcal{C}$ on $\mathcal{S}_{0}$ ).

Then the data $\bar{\phi}, \overline{\boldsymbol{\mu}_{i}}, \overline{\dot{\phi}}$ and $\overline{\dot{\boldsymbol{\mu}}_{j}}, i, j=1,2,3$, on the surface $\mathcal{S}_{1}$ fulfill the conditions $\mathbf{S} 1$ through $\mathbf{S} 4$ of theorem $\mathbf{2}$. Hence, functions $\phi$ and $\boldsymbol{\mu}_{i}$ on a 3 dimensional neighbourhood $\mathcal{U}$ of $\mathcal{S}_{1}$ can be obtained such that (11) and (17) are fulfilled.

Therefore the metric $\tilde{g}$ obtained by substituting these $\phi$ and $\boldsymbol{\mu}_{i}$ in equation (2) has constant curvature.

\section{Two examples}

For the sake of illustration we shall consider two cases of 3-dimensional Riemannian manifolds and locally deform them into flat metrics, in the sense stated in Theorem 2. Since both cases exhibit some symmetries, the solutions will be rather proposed than derived by solving the partial differential system presented in sections 2 and 3. 


\subsection{Schwarzschild space}

The title is a shortening for the space 3-manifold for Schwarzschild coordinates in Schwarzschild spacetime. The metric is:

$$
\hat{g}=\kappa^{-1} d r \otimes d r+r^{2} d \theta \otimes d \theta+r^{2} \sin ^{2} \theta d \varphi \otimes d \varphi
$$

with $\kappa=1-\frac{2 m}{r}$, in the region $r>2 m$ (otherwise the metric is not Riemannian).

This metric can be deformed into a flat metric in several ways. Among others:

4.1. A Choosing $s=\sqrt{\kappa^{-1}-1} d r$, we readily obtain:

$$
\hat{g}=\tilde{g}+s \otimes s
$$

where $\tilde{g}=d r \otimes d r+r^{2} d \theta \otimes d \theta+r^{2} \sin ^{2} \theta d \varphi \otimes d \varphi$ is flat.

4.1.B It is well known tha,t changing $r$ into the coordinate

$$
R=\frac{1}{2}(r \sqrt{\kappa}+r-m) \quad r=R\left(1+\frac{m}{2 R}\right)^{2},
$$

the metric becomes: $\hat{g}=\sigma \tilde{g}$, where

$$
\sigma=\left(1+\frac{m}{2 R}\right)^{4} \quad \text { and } \quad \tilde{g}:=d R \otimes d R+R^{2} d \theta \otimes d \theta+R^{2} \sin ^{2} \theta d \varphi \otimes d \varphi
$$

is a flat metric.

\subsection{Kerr space}

In Boyer-Lindquist coordinates, the Kerr metric is [14]:

$$
g=-\frac{\Delta}{p^{2}}\left[d t-a \sin ^{2} \theta d \varphi\right]^{2}+\frac{\sin ^{2} \theta}{p^{2}}\left[a d t-\left(r^{2}+a^{2}\right) d \varphi\right]^{2}+\frac{p^{2}}{\Delta} d r^{2}+p^{2} d \theta^{2}
$$

where $p^{2}=r^{2}+a^{2} \cos ^{2} \theta$ and $\Delta=r^{2}-2 m r+a^{2}$.

The stationary space 3-manifold is endowed with the Riemannian metric:

$$
\hat{g}=\frac{p^{2}}{\Delta} d r \otimes d r+p^{2} d \theta \otimes d \theta+\frac{p^{2} \Delta}{p^{2}-2 m r} \sin ^{2} \theta d \varphi \otimes d \varphi
$$

or, equivalently,

$$
\hat{g}=\frac{p^{2}}{r^{2}}\left(\frac{1}{\Delta} d r \otimes d r+r^{2} d \theta \otimes d \theta+\frac{\Delta}{p^{2}-2 m r} r^{2} \sin ^{2} \theta d \varphi \otimes d \varphi\right)
$$

in the region $r^{2}-2 m r+a^{2} \cos ^{2} \theta>0$ (otherwise the metric is not Riemannian). Similarly as in the case $\mathbf{B}$ above, this last expression suggests to define:

$$
R=\frac{1}{2}(\sqrt{\Delta}+r-m) \quad r=R\left[\left(1+\frac{m}{2 R}\right)^{2}-\frac{a^{2}}{4 R^{2}}\right]
$$


Then (32 can be written as:

$$
\hat{g}=\frac{p^{2}}{R^{2}}\left(\tilde{g}+\frac{a^{2} R^{2} \sin ^{4} \theta}{p^{2}-2 m r} d \varphi^{2}\right)
$$

where $\tilde{g}=d R \otimes d R+R^{2} d \theta \otimes d \theta+R^{2} \sin ^{2} \theta d \varphi \otimes d \varphi$, which has already the form (11) with $\epsilon=+1$ and

$$
\sigma=\frac{p^{2}}{R^{2}} \quad \text { and } \quad s=\frac{a p \sin ^{2} \theta}{\sqrt{p^{2}-2 m r}} d \varphi
$$

\section{Conclusion and outlook}

We have shown that, locally, any Riemannian 3-dimensional metric $g$ can be deformed along a direction $s$ into a metric $\sigma h$ that is conformal to a metric of constant curvature, as stated in theorem 1.

The direction $s$ is not uniquely determined by the metric $g$, and the decomposition (1) can be achieved in an infinite number of ways. Determining more precisely the class of $\sigma$ and $s$ which deform a given $g$ into a constant curvature metric $h$ will be the object of future work. Specially the case where both, $g$ and $h$, are flat.

It is tempting to look at (11) as an equivalence relation, namely,

$$
g_{1} \sim g_{2} \text { if, and only if, } \exists \sigma \text { and } s \text { such that: } g_{1}=\sigma g_{2}+\epsilon s \otimes s .
$$

However, this relation is not in general transitive.

Expression (1) can also be taken as the definition of a transformation of a metric $h$ into a metric $g$, somewhat reminding of Kerr-Schild transformations [15]. Namely, given $\sigma \in \Lambda^{0}(\mathcal{V}), \epsilon= \pm 1$ and $s \in \Lambda^{1}(\mathcal{V})$ we can define the transformation:

$$
T_{\sigma, \epsilon, s}: g_{1} \longmapsto g_{2}=T_{\sigma, \epsilon, s}\left[g_{1}\right]=\sigma g_{1}+\epsilon s \otimes s .
$$

which acts on Riemannian metrics on the 3 -manifold $\mathcal{V}$. It is obvious that the identity and the inverse of $T$ belong to this class of transformations, but the product $T_{\sigma, \epsilon, s} \circ T_{\sigma^{\prime}, \epsilon^{\prime}, s^{\prime}}$ do not in general belong to this class. One could ask however whether this class contains some groups of transformations.

Finally, it would be interesting to extend theorem 1, or a similar result, to a higher number of dimensions. In this sense it seems worth to pursue the conjecture of universal deformation law [5], namely, any Riemannian (resp., semi-Riemannian) metric $g$ can be written as:

$$
g_{\alpha \beta}=\sigma h_{\alpha \beta}+\mu F_{\alpha \lambda} F_{\beta}^{\lambda}
$$

where $F_{\alpha \beta}$ is a 2-form, $h$ is a flat metric and $\sigma$ and $\mu$ are scalar functions of $F$. (Actually, expression (11) is a particular case of the above conjecture for $n=3$, with $F_{i j}=\eta_{i j k} s^{k}$, for $i, j, k=1,2,3$. 


\section{Acknowledgement}

The work of J. Ll. and of D. S. is supported by DIGICyT, contract no. BFM2000-0604 and by Institut d'Estudis Catalans (S.C.F.).

\section{Appendix A: The volume tensors}

Let us choose be a $\tilde{g}$-orthonormal frame, $\left\{\tilde{e}_{i}\right\}_{i=1,2,3}$, such that $M_{i j}=A \delta_{i}^{3} \delta_{j}^{3}$. From (2) we have that:

$$
g_{i j}=\phi^{-1}\left(\delta_{i j}+A \delta_{i}^{3} \delta_{j}^{3}\right), \quad \tilde{g}_{i j}=\delta_{i j}
$$

whence it follows that

$$
D^{2}:=\frac{\operatorname{det} g}{\operatorname{det} \tilde{g}}=\phi^{-3}(1+A)
$$

Now, $A$ is related to the invariant $m_{0}:=g^{i j} M_{i j}$. Indeed,

$$
m_{0}=g^{33} A=\frac{\phi}{1+A} A
$$

that yields $A=m_{0}\left(\phi-m_{0}\right)^{-1}$, which substituted into (35) yields:

$$
D^{2}=\frac{\phi^{-2}}{\phi-m_{0}}
$$

Now, let $\left\{\tilde{\omega}^{j}\right\}_{j=1,2,3}$ be the dual basis. The contravariant volume tensors for $\tilde{g}$ and $g$ are, respectively,

$$
\tilde{\eta}^{i k l}=\epsilon^{i k l} \quad \text { and } \quad \eta^{i k l}=D^{-1} \epsilon^{i k l}
$$

where $\epsilon^{i k l}$ is the Levi-Civittà symbol. From the latter it follows immediately that:

$$
\tilde{\eta}^{i k l}=D \eta^{i k l},
$$

Since both, $g$ and $\tilde{g}$, are Riemannian, i. e., non-degenerate and positive, it turns out that $\phi>0$ and $D^{2}>0$, which, taking (36) into account, amounts to:

$$
\phi>0 \quad \text { and } \quad \phi>m_{0}
$$

\section{References}

[1] RIEMAnN, G. F. B., Über die Hypothesen, welche der Geometrie zu Grunde liegen, Abhand. K. Ges. Wiss. Göttingen, 13, 133, 1868; English translation by W.K. Clifford, Nature 8, 14, 1873; reprinted and edited by H. Weyl, Springer, Berlin, 1920. Included in its Gesammelte Mathematische Werke, wissenschaftlicher Nachlaß und Nachträge, eds. H. Weber, R. Dedekind, B.G. Teubner, Leipzig, 1892; 2d ed. Dover Publ., New York, 1953. 
[2] Roughly speaking, a geometric object is a mathematical object which remains unchanged under changes of local charts.

[3] EISENHART, L. P., A Treatise on the Differential Geometry of Curves and Surfaces, Dover Publications, Inc. New York, 1960, p 93.

[4] In spite of its interest, the known results on diagonalization of threedimensional metrics do not belong to this type: in all of them, in addition to the $f=3$ scalars, an ortogonal tetrad is also involved with the (more o less implicit) flat metric. See CARTAN, E., Les systèmes differentiels extérieurs et leurs applications géométriques, Hermann, Paris, 1945 and 1971 for an analytic proof on the existence of orthogonal coordinates; DETURCK, M., Duke Math. Jour., 51, p 243-60, 1984 for a $C^{\infty}$ proof; WALBERER, P., Abhandl. Math.Sem. Univ. Hamburg, 10, p 169-79, 1934 for the decomposition in a given orthogonal frame; BEL, L., Gen. Relat. and Grav., 28, p 1139-50, 1996 for decompositions in principal frames; TANNO, S., J. Differential Geometry, 11, p 467-74, 1976 for other particular decompositions.

[5] In the context of the General Theory of Relativity, such a n-dimensional relation has been proposed by one of us, but unfortunately it remains for the moment only a mere conjecture. See COLL, B., A Universal Law of Gravitational Deformation for General Relativity, in Proceedings of the Spanish Relativity Meeting in honour of the 65th Birthday of Lluis Bel "Gravitation and Relativity in General" ed. by J. Martin, E. Ruiz, F. Atrio, A. Molina, World Scientific, 1999.

[6] See for example O'NEILL, B., Elementary Differential Geometry, Academic Press, 1966.

[7] In this case, the deformation of material coordinates does not change the flat character of the metric, only its tensor expression (Saint Venant equations).

[8] LiCHNEROWICZ, A., J. Math. Pures Appl. 23, p 37-63, 1944.

[9] We shall use the definition of the curvature tensor in terms of a general basis given in: CHOQUET-BRUHAT, Y., DEWITT-MORETTE, C. and DILLARD-BLEICK, M., Analysis, Manifolds and Physics, p. 306, revised edition, North-Holland (Amsterdam, 1987). Nevertheless, we shall write the first pair of indices in the positions $R_{j k l}^{i}$ as in EISENHART, L. P., Riemannian Geometry, eq. (8.3), Princeton University Press (Princeton, N.J. 1997).

[10] EISENHART, L. P., Op. cit., eq. (28.12),

[11] EISENHART, L. P., Op. cit., p. 83,

[12] EISENHART, L. P., Op. cit., eq. (26.2) Princeton University Press (Princeton, N.J. 1997) 
[13] JOHN, F., Partial Differential Equations, Chap. 3, Springer (New York, 1971)

[14] MiSNER, C. W., THORNE, K. S. and WHEELER, J. A., Gravitation, p. 877, Freeman, San Francisco (1973)

[15] KRAMERS, D., STEPHANI, H., HERLT, E. and McCALLUM, M., Exact solutions of Einstein's field equations, p. 299, Cambridge University Press, Cambridge (1979) 